\title{
COOPERATIVISMO, FACTOR EMPRESARIAL Y DESARROLLO ECONÓMICO: PROPUESTA DE UN MODELO TEÓRICO DE ENLACE
}

\author{
POR \\ Carmen GUZMÁN ALFONSO ${ }^{1}$, \\ Francisco J. SANTOS CUMPLIDO ${ }^{2}$ y \\ María de la O BARROSO GONZÁLEZ ${ }^{3}$
}

\section{RESUMEN}

Dados sus principios de funcionamiento, las cooperativas son consideradas por diversos estudios como una fórmula empresarial que puede contribuir de forma destacada a superar la crisis económica iniciada en 2008. Asimismo, existen numerosas investigaciones que señalan que estas entidades juegan además un papel muy relevante en el desarrollo económico de cualquier territorio. No obstante, cuando se examina el conjunto de la literatura económica sobre cooperativas se observa que éstas son estudiadas en la mayoría de los casos a partir de sus propias particularidades y características de participación y solidaridad. En este sentido, siguiendo un marco de análisis diferente, este trabajo se basa en la teoría del entrepreneurship o factor empresarial para proponer un modelo teórico que explique el comportamiento de este tipo de organización empresarial y, más concretamente, como ese comportamiento puede contribuir al desarrollo económico de los territorios.

Palabras clave: cooperativas, emprendimiento, empresas, desarrollo económico, esencia cooperativa

Claves Econlit: A130; L26; O11; P130

\footnotetext{
1 Universidad de Sevilla. Departamento de Economía Aplicada I. Dirección de correo electrónico: cguzman2@us.es.

${ }^{2}$ Universidad de Sevilla. Dirección de correo electrónico: fjsantos@us.es.

${ }^{3}$ Universidad de Huelva. Dirección de correo electrónico: barroso@uhu.es.

REVESCO No 122 - Tercer Cuatrimestre 2016 - ISSN: 1885-8031 - www.ucm.es/info/revesco

http://dx.doi.org/10.5209/rev_REVE.2016.v122.52018
}

Fecha de recepción: 27/04/2015

Fecha de aceptación: 21/12/2015 


\title{
COOPERATIVISM, ENTREPRENEURSHIP AND ECONOMIC DEVELOPMENT: A LINKING THEORETICAL MODEL
}

\begin{abstract}
Cooperatives, as a kind of firms, are considered by many scholars as an remarkable alternative for overcoming the economic crisis started in 2008. Besides, there are other scholars which pointed out the important role that these firms play in the regional economic development. Nevertheless, when one examines the economic literature on cooperatives, it is detected that this kind of firms is mainly studied starting from the point of view of their own characteristics and particularities of participation and solidarity. In this sense, following a different analysis framework, this article proposes a theoretical model in order to explain the behavior of cooperatives based on the entrepreneurship theory with the aim of increasing the knowledge about this kind of firms and, more specifically, their contribution to regional economic development.
\end{abstract}

Keywords: cooperatives, entrepreneurship, entreprises, economic development, cooperative essence.

\section{INTRODUCCIÓN}

A lo largo de los últimos años, se ha producido un considerable aumento del interés por parte de los poderes públicos e investigadores respecto a las cooperativas. Ese interés se debe fundamentalmente a que se cuenta con suficientes evidencias para afirmar que las cooperativas han demostrado resistir mejor la crisis económica iniciada en 2008 que las empresas típicamente capitalistas (Birchall, 2013; Cantero, González Loureiro y Puig, 2013). De hecho, en España la destrucción de empleo en las cooperativas ha sido siete puntos y medio inferior a la del conjunto de las fórmulas empresariales (Ministerio Empleo y Economía Social, 2013).

Por otro lado, en cuanto al aumento del interés por parte de los investigadores, cabe señalar que es consecuencia de que las cooperativas son percibidas por varios académicos también como la forma más adecuada para salir de la actual crisis económica (Melián y Campos, 2010; Monzón y Chaves, 2012). Sus argumentos se basan en que las imperfecciones del sistema económico capitalista, aquéllas que han conducido a la crisis económica internacional, no se reproducen en estas entidades de economía social ya que éstas se guían 
por valores y principios diferentes (Vázquez, Albarrán y Salinas, 2013; Santos, Barroso y Guzmán, 2013).

Cabe destacar que el estudio de las cooperativas no es algo nuevo en la literatura económica. Esta forma empresarial es objeto de gran interés por parte de los investigadores a partir de la segunda mitad del siglo XX (Ward, 1958) y, especialmente, desde los años ochenta. Su objetivo ha sido analizar a través de la teoría neoclásica a las empresas cuya propiedad pertenecía a los trabajadores de las mismas, también llamadas "empresas autogestionadas". Estos investigadores realizaron varios trabajos empíricos con el fin de comprobar si la participación de los trabajadores tanto en la propiedad de la empresa como en el reparto de beneficios y en la toma de decisiones afectaba de alguna manera a su desempeño (Jones y Svenjar, 1982; Kruse y Blasi, 1997). Asimismo, también se realizaron trabajos destinados a comprobar si existían diferencias de productividad con las empresas capitalistas, no siendo la evidencia empírica concluyente (Lee, 1988; Jones, 2007).

También a partir de los años ochenta empezaron a surgir estudios que tenían por objeto definir el papel de las cooperativas dentro de los procesos de desarrollo económico. Dichos estudios analizaban cómo los principios de compromiso con el entorno y los valores de solidaridad y reciprocidad arraigaban estas entidades a sus territorios y generaban efectos de arrastre en los mismos (Ben-Ner, 1988b; Westlund y Westerdahl, 1996). Dicha línea de investigación se ha seguido desarrollando desde entonces y, en la actualidad, supone una de las principales líneas de investigación dentro del campo de estudio de estas entidades (Chaves y Monzón, 2012; Martínez Charterina, 2015).

Por tanto, las cooperativas han sido objeto de atención por parte de numerosos investigadores debido tanto a las particularidades que las definen como a su contribución al desarrollo económico. Sin embargo, cabe señalar que a lo largo de las cuatro últimas décadas, de forma simultánea al estudio de las cooperativas, ha surgido un nuevo ámbito de estudio que ha adquirido cierta relevancia en la ciencia económica, el del factor empresarial o entrepreneurship, cuya aplicación al análisis del comportamiento de las cooperativas podría ayudar a conocer mejor a estas entidades y su posible contribución al desarrollo económico.

La línea de investigación en entrepreneurship considera que la actividad empresarial desempeña un papel fundamental en el proceso de desarrollo económico de los territorios (Hébert y Link 1989; Guzmán, 1994; Wennekers y Thurik, 1999; Santos, 2004; Fernández y 
Romero, 2013). Esta relación entre actividad empresarial y desarrollo económico se basa en que los empresarios y las empresas son considerados los agentes responsables de crear riqueza y empleo a la vez que asumen los retos que la globalización impone, tales como la fragmentación de mercados o el aumento de la competencia y la incertidumbre (Carlsson, 1996; Santos, 2004).

Desde los años setenta, se han llevado a cabo iniciativas destinadas a promover el espíritu emprendedor y a fomentar la creación de empresas como estrategia básica para dinamizar las economías. Entre los objetivos de estas iniciativas destaca la necesidad de flexibilizar el tejido productivo para acelerar el cambio estructural y, en definitiva, para avanzar hacia el desarrollo económico a largo plazo. Estas iniciativas se han fomentado desde diversos organismos internacionales, los cuales han recomendado la puesta en marcha de políticas de promoción de pequeñas y medianas empresas para el desarrollo de los territorios (OCDE, 1998, 2003). En España, la iniciativa más reciente y de mayor calado ha sido la aprobación por el Gobierno del país de la Ley 14/2013 de 27 de septiembre de apoyo a los emprendedores y su internacionalización, más conocida como "Ley de emprendedores", en la cual se recogen diversas medidas para facilitar la creación de nuevas empresas.

Así pues, teniendo en cuenta a) la importancia que tiene la perspectiva del entrepreneurship en economía, y b) que su aplicación sobre el factor empresarial cooperativo podría ayudar a conocer mejor las características de estas entidades y sus posibilidades de contribución al desarrollo económico, el objetivo de este trabajo consiste en proponer un modelo teórico de enlace entre ambas líneas de investigación con el fin último de abrir nuevos cauces para investigaciones futuras sobre cooperativas. Para ello, se realizará, en primer lugar, un análisis de las características de las cooperativas y sus vínculos con el desarrollo económico. En segundo lugar, se establecerán las dimensiones que definen el entrepreneurship desde una perspectiva macroeconómica. Por último, se propondrá el modelo de enlace entre ambas líneas de investigación.

\section{LAS COOPERATIVAS Y EL DESARROLLO ECONÓMICO}

\subsection{Cooperativismo y desarrollo local: similitudes}

El rol que ostentan las cooperativas en los procesos de desarrollo local ha sido objeto de atención por parte de numerosos autores durante las cuatro últimas décadas (McRobie, 1981; Mactchaya y Pérotin, 2013). Este reconocimiento por parte de los investigadores y 
políticos sobre el papel que ejercen las cooperativas en el desarrollo económico está unido a la aceptación general que desde los años ochenta se ha concedido al modelo de desarrollo local y sostenible (Pastor, 2011).

El desarrollo local como modelo de desarrollo empezó a ser puesto en práctica en los años ochenta del siglo XX por los gobiernos locales con el objetivo de hacer frente a las graves consecuencias que la globalización empezaba a provocar. En lugar de que el desarrollo económico fuese generado por fuerzas externas -es decir, por empresas exteriores a la zona establecidas en municipios con factores productivos más baratos que en el lugar de origen- se empezó a considerar la idea de llevar a cabo un desarrollo generado por fuerzas endógenas, entendiendo por éstas actuaciones que emanasen del territorio y que aprovechasen los recursos propios para aumentar la competitividad, el empleo y la calidad de vida de la comunidad (Gómez y Román, 2005). Es decir, durante los ochenta se pasó de una perspectiva de desarrollo "top-down" a una de desarrollo "bottom-up", lo cual implicaba un cambio estructural del sistema socioeconómico. De esta manera, en este nuevo modelo se pretendía que no sólo las empresas locales participaran en este proceso de desarrollo sino también las instituciones y la población local, ya que todos eran responsables de las inversiones y de la toma de decisiones concernientes al desarrollo (Stöhr, 1984).

Por su parte, también en estos años empieza a fraguarse el concepto de desarrollo sostenible. Éste surge como consecuencia del daño que el crecimiento económico causa sobre el medio ambiente. El Informe Bruntland (Comisión Mundial de Medio Ambiente y Desarrollo, 1987) es el más referenciado a este respecto y define el desarrollo sostenible de la siguiente manera: "el desarrollo sostenible es aquel que permite satisfacer las necesidades de las generaciones presentes sin comprometer las posibilidades de las del futuro para atender a sus propias necesidades". Esta definición sustenta el concepto de desarrollo sobre tres pilares: el económico, el social y el medioambiental; lo cual conlleva conceder una prioridad al medioambiente y a la sociedad en los procesos de desarrollo que hasta entonces había sido inexistente.

En este sentido, parece que se podría afirmar que la filosofía cooperativa coincide con la estrategia de desarrollo local y sostenible. Esta vinculación se debe principalmente a que en el cooperativismo los individuos participan en un proyecto que va más allá del ámbito individual, lo cual tiene lugar a través de los principios de funcionamiento cooperativos (Puentes y Velasco, 2009). Por ejemplo, según el principio democrático, los miembros 
participan a través del voto en todas las decisiones que afectan no sólo a ellos a título personal, sino a la cooperativa, entendiendo ésta como un grupo de personas (Pastor, 2011).

Asimismo, los principios de educación, compromiso con la comunidad y cooperación muestran que la actividad cooperativa no sólo tiene como objetivo repercutir sobre los integrantes de la entidad sino sobre el resto de la población (Carrasco, 2005; Martínez Charterina, 2015). De este modo, en base al principio de educación y promoción, los miembros tienen la misión de fomentar al público en general los beneficios del cooperativismo, además de ofrecer la oportunidad de incrementar su formación (ACI, 1995).

Por otro lado, según el principio de compromiso con la comunidad, la cooperativa no sólo se preocupa por los intereses de sus miembros, sino también de los demás grupos de interés del territorio, tales como los desempleados, los proveedores, los clientes y la sociedad en general. También según el principio de compromiso, la cooperativa se preocupa del medioambiente que rodea a esos grupos de interés, teniéndolos a todos en cuenta a la hora de desarrollar su actividad económica (Carrasco, 2005; Mozas y Bernal, 2006; Saz y Carús, 2008; Puentes y Velasco, 2009). Además de ello, el fuerte arraigo al territorio les lleva a servirse de los recursos naturales, económicos y humanos de la zona, generando efectos de arrastre al igual que cualquier estrategia de desarrollo local (Tarazona y Albors, 2005; Calvo y González, 2011).

Por último, en relación al principio de cooperación, los miembros de una cooperativa tienen la obligación de llevar a cabo la cooperación con otras cooperativas y otras entidades por medio de estructuras locales, nacionales, regionales e internacionales con el objetivo de fortalecer el desarrollo de la empresa y el movimiento cooperativo. De esta manera, el principio de cooperación promueve la creación de partenariados con otras cooperativas, autoridades locales, sindicatos, etc; aunque sea de manera informal (Buendía y Carrasco, 2014). Estas relaciones contribuirían así a la construcción de una red de capital social con todos los beneficios que ello conlleva, incluyendo la cohesión social y la transmisión de conocimiento (Pastor, 2011). De esta forma, se generaría un efecto multiplicador de sus objetivos y filosofía y la creación de nuevas actividades económicas y empleos (Tarazona y Albors, 2005; Calvo y González, 2011). En definitiva, el cooperativismo sería un modelo que, al igual que el desarrollo endógeno, se comporta siguiendo una forma de actuar de abajo hacia arriba (bottom-up), donde la sociedad local es capaz de dinamizar la economía y mejorar la calidad de vida de la población (Coll y Cuñat, 2007). 
Sirva de ejemplo el caso de Mondragón, que empezó en 1943 con la creación por parte de José María de Arizmendiarrieta de la Escuela Profesional, la cual era democráticamente administrada y estaba abierta a todos los jóvenes de la comarca. De esta escuela salió la primera cooperativa de la zona en 1956, ULGOR (hoy Fagor Electrodomésticos), formada por cinco antiguos alumnos y dedicada a fabricar estufas y cocinas de petróleo. Las relaciones de dicha cooperativa con otras entidades generaron un sinfín de nuevas actividades económicas y de nuevos empleos 5 . En este sentido, se puede afirmar que las cooperativas contemplan las relaciones sociales y la implicación de la ciudadanía para influir directamente en la situación económica y social del territorio, al igual que se hace con la estrategia de desarrollo local (Coll y Cuñat, 2007).

\subsection{Otros factores de las cooperativas que favorecen el desarrollo local}

Al margen de las coincidencias entre el cooperativismo y el desarrollo local a través de los principios cooperativos, existen otros factores en estas entidades que, por su naturaleza, favorecen la cohesión social y el desarrollo de los territorios. Para empezar, su razón de ser reside en cubrir determinados servicios que el sector privado y público no proporcionan en calidad, cantidad o precios adecuados (Buendía y García, 2003). Por lo tanto, sin su existencia, dichas necesidades seguirían sin estar cubiertas.

Además de ello, en base a la literatura existente se ha demostrado que las cooperativas generan empleos estables y de calidad, fieles a sus valores propios (Mozas y Bernal, 2006; Calderón y Calderón, 2012a). Asimismo, la seguridad en el empleo, las relaciones interpersonales o la retribución que las cooperativas ofrecen, generan un aumento de la satisfacción laboral de sus integrantes (Gargallo y Freundlich, 2010; Marcuello y Nachar, 2013). De igual modo, se ha demostrado que la participación en la toma de decisiones también afecta positivamente sobre la satisfacción laboral de los miembros de estas entidades (Miller y Monge, 1986).

En este contexto, cabe destacar que son varios los autores que encuentran que las cooperativas son las organizaciones idóneas sobre las que basar los procesos de desarrollo local, especialmente en momentos de crisis, debido a la manera que tienen de responder ante los cambios en el mercado. Dichas respuestas se refieren, por ejemplo, a comportamientos

\footnotetext{
${ }^{5}$ Cabe señalar que actualmente esta entidad es objeto de muchas críticas como consecuencia de su estrategia empresarial, la cual ha incluido decisiones de deslocalización de su actividad que han desvirtuado en cierta manera su modelo cooperativo (Mendizábal et al., 2005; Errasti y Mendizábal 2007).
} 
que conllevan la internalización de externalidades negativas del mercado por parte de las cooperativas, reaccionando con el descenso de jornada laboral o salarios en lugar de proceder a una reducción de plantilla (Burdin y Dean, 2009; Jones y Kalmi, 2009; Santos et al., 2013).

Asimismo, la literatura también destaca el carácter contracíclico de las cooperativas (Ben-Ner, 1988b). En este sentido, existen algunos estudios que comparan el ciclo económico con la evolución de estas entidades a través de determinados indicadores de empleo (Defourny 1990; Cornforth y Thomas, 1995, Calderon y Calderón 2012b). Estos estudios encuentran que durante las crisis el empleo en las empresas convencionales disminuye en mayor medida que en las cooperativas, habiendo incluso casos en los que el empleo en las cooperativas aumenta.

Por último, es necesario reconocer que las cooperativas son empresas que compiten con las empresas tradicionales, por lo que para seguir existiendo no tendrán más remedio que ser eficientes y competitivas, consolidando así el empleo creado y contribuyendo a aumentar el crecimiento económico local. Según Monzón (2010), el valor añadido bruto de las cooperativas en España en 2008 fue de poco más de 10.400 millones de euros (sobre 8.610 millones de euros sin contar a las cooperativas de crédito). En dicho año se contabilizaban 24.738 cooperativas, las cuales creaban 456.870 empleos directos y facturaron casi 66.300 millones de euros. Por su parte, a nivel internacional, el CIRIEC señalaba en su comunicación al Comité Económico y Social Europeo de 2012 que las cooperativas de la UE-27 suponían un 2,1\% del empleo total del territorio durante los años 2009-2010. Asimismo, reconocía un aumento de un $31,51 \%$ de los empleos remunerados en las cooperativas desde 2002 hasta 2010, teniendo en cuenta sólo la UE-15 (Monzón y Chaves, 2012).

En definitiva, la naturaleza de las cooperativas, los principios y valores por los que éstas se rigen, y el carácter empresarial de las mismas hacen que a éstas se les asigne un rol clave en las estrategias de desarrollo endógeno (Coque, 2008; Puentes y Velasco, 2009). Prueba de ello es que el Parlamento Europeo aprobó el 2 de julio de 2013 un informe en el cual reconocen que las cooperativas, dadas sus particularidades, son las entidades más adecuadas para salir de la crisis iniciada en 2008. En consecuencia, el Parlamento Europeo exige a la Comisión que refuerce las iniciativas destinadas a la promoción de las mismas (Parlamento Europeo, 2013). 


\section{EL FACTOR EMPRESARIAL O ENTREPRENEURSHIP}

\subsection{Delimitación del concepto de factor empresarial}

A pesar de que aún no existe en la literatura económica una definición generalmente aceptada sobre el factor empresarial (Baumgartner, Schulz y Seidl, 2013), la comunidad científica coincide en que el fenómeno del entrepreneurship engloba tanto a la persona de la que parte la iniciativa empresarial, como a la empresa propiamente dicha en la cual se materializa dicha iniciativa empresarial. En este sentido, se puede afirmar que el factor empresarial se compone de dos grandes dimensiones: el empresario o emprendedor y la empresa (Guzmán y Santos, 2009).

Siguiendo a Hébert y Link (1989), podemos afirmar que el empresario "es alguien que se especializa en tomar decisiones de manera juiciosa sobre la distribución, la forma y el uso de bienes, recursos e instituciones, responsabilizándose de los resultados de dichas decisiones" (Hébert y Link, 1989). Por su parte, la empresa es definida por Plaza-Angulo de la siguiente manera: "una organización dotada de una estructura interna propia que se erige como un sistema social autónomo que actúa, dentro de un determinado contexto social, político y económico, como una unidad de producción de bienes y servicios con el fin de alcanzar unos objetivos concretos" (Plaza-Angulo, 2010).

Ante tales definiciones y en la medida en que una cooperativa produce un producto o servicio y sus miembros toman decisiones sobre dicha producción, se puede afirmar que una cooperativa es un tipo específico de empresa que está formada por emprendedores. En este sentido, las cooperativas de un territorio constituirían lo que podemos llamar como el "factor empresarial cooperativo" del territorio.

\subsection{La contribución del factor empresarial al desarrollo económico}

El papel del factor empresarial en el desarrollo económico ha sido considerado a lo largo de toda la historia del pensamiento económico por diferentes autores (Schumpeter, 1934; Baumol, 1990). De entre todas las contribuciones, el profesor Guzmán (1994) concluye tras realizar una síntesis de teorías que el rol que el factor empresarial desempeña en el proceso de desarrollo económico de un territorio estará determinado tanto por la cantidad de factor empresarial con el que cuente dicho territorio (vertiente cuantitativa del factor empresarial), como por la calidad del factor empresarial de la zona (vertiente cualitativa de 
factor empresarial) (Fernández y Romero, 2013). De este modo, a partir de la literatura sobre entrepreneurship y desarrollo económico se entiende que, a mayor cantidad de factor empresarial, mayor desarrollo económico en el territorio.

Cabe señalar que cuando la literatura económica ha tratado de explicar el desarrollo económico, no siempre ha tenido en cuenta las dos perspectivas (cuantitativa y cualitativa) de análisis del factor empresarial. De hecho, tradicionalmente se ha vinculado al factor empresarial con el desarrollo económico desde una vertiente cuantitativa (OCDE, 1998). No obstante, a medida que avanzaban las investigaciones se empezó a reconocer que no todos los empresarios y empresas contribuían de la misma forma al proceso de crecimiento y desarrollo económico, pues no todos generaban el mismo número de empleos ni contribuían en igual medida al crecimiento del Producto Interior Bruto (Baumol, 1990).

Por ello, además del número de empresas y empresarios existente en un territorio, se empieza a aceptar la idea de que determinados aspectos cualitativos de los mismos también son relevantes para el desarrollo económico (Guzmán y Santos, 2009). Por tanto, cuando se estudia la influencia del factor empresarial en el desarrollo económico se puede afirmar que no basta sólo con considerar la "cantidad”, sino también la "calidad” del mismo. Así, Guzmán (2006) sostiene que la cantidad de factor empresarial es una condición necesaria pero no suficiente para el desarrollo económico. En esta línea, existen algunos trabajos en los que se ponen de manifiesto de forma expresa la relación entre el grado de desarrollo de un territorio y la calidad de su factor empresarial (Santos et al. 2012; Fernández y Romero 2013).

\subsection{La calidad macroeconómica del factor empresarial}

Como hemos visto que el factor empresarial engloba tanto la dimensión del empresario o emprendedor como la dimensión de la empresa, se puede afirmar que para que el factor empresarial de un territorio sea de calidad, los empresarios y las empresas (en concreto, la estructura empresarial desde una perspectiva macroeconómica) de dicho territorio también deben ser de calidad. A continuación examinamos las variables que determinan que un factor empresarial sea de calidad, diferenciando entre la perspectiva del empresario y de la empresa. 


\subsubsection{Desde la perspectiva del empresario}

La calidad del factor empresarial desde la perspectiva del empresario o emprendedor empresarial se determina a través de una serie de comportamientos (Santos et al., 2012). Todos esos comportamientos se manifiestan a su vez en la empresa en la que el empresario realiza sus funciones, pues ésta es el vehículo sobre el que los individuos transforman sus cualidades en acciones (Wennekers y Thurik, 1999).

Tras un análisis de la literatura sobre los comportamientos emprendedores, se puede afirmar que las principales dimensiones o comportamientos que definen la calidad del empresario son la innovación, la asunción de riesgos, la proactividad, la ambición y la cooperación. Todos estos comportamientos influirían positivamente sobre el desempeño y crecimiento de la empresa, lo cual podría repercutir a su vez sobre el desarrollo económico del territorio (Rauch et al., 2005).

En primer lugar, la innovación es el comportamiento empresarial que lleva a la empresa a implicarse en procesos creativos y de experimentación y generación de nuevas ideas, los cuales conducen a su vez a nuevos productos, servicios y procesos tecnológicos o de gestión (Lumpkin y Dess, 1996; Schumpeter, 1934). Esta dimensión del comportamiento emprendedor ha sido fundamental desde que Schumpeter (1934) resaltara el papel de la innovación en los procesos empresariales y de crecimiento y desarrollo económico.

En segundo lugar, la asunción de riesgos se ha considerado un comportamiento ligado al emprendedor desde los primeros trabajos del economista Cantillón a principios del siglo XVIII. En tiempos recientes, la literatura específica sobre entrepreneurship ha seguido relacionando el comportamiento emprendedor con determinados niveles de riesgos (Miller, 1983; Covin y Slevin, 1989). La asunción de riesgos tiene lugar cuando una persona se involucra en una situación que puede reportar beneficiosas recompensas en caso de éxito, pero también severas consecuencias si el individuo fracasa (Miller, 1983).

En tercer lugar, la proactividad es el comportamiento en virtud del cual el empresario busca nuevas oportunidades en el mercado para actuar de forma anticipada a los cambios de demanda (Venkataraman, 1989). Así, los empresarios proactivos pueden ser los primeros en entrar en los nuevos mercados o también los más rápidos a la hora de introducir mejoras. Todo ello influirá de forma muy positiva en los resultados de la empresa, en el desempeño de la misma y en su posición en el mercado (Penrose, 1959). 
En cuarto lugar, la ambición puede ser definida como la necesidad que tiene el empresario de seguir desarrollando y ampliando su negocio de forma continuada (Guzmán y Santos, 2009). Esta necesidad se muestra a través del crecimiento de la empresa y se contrapone al sentimiento de conformidad que tienen los empresarios una vez que el negocio ha alcanzado cierto tamaño (Davidsson, 1991).

En quinto lugar, la cooperación es el comportamiento emprendedor que implica trabajar conjuntamente con otros empresarios y empresas o instituciones con la intención de lograr mayores cotas de crecimiento y reforzar la posición competitiva de la empresa en el mercado (Guzmán y Santos, 2009). Aunque tradicionalmente la cooperación no se ha considerado un elemento determinante a la hora de definir la calidad del comportamiento emprendedor, desde hace algunas décadas esta tendencia está cambiando (Doh y McNeely, 2012). La importancia de la cooperación radica en que ésta contribuye a reforzar la posición competitiva de las empresas y, consecuentemente, de sus territorios. La manera en que se consigue esta mejora competitiva es generando una serie de beneficios a la actividad de la empresa, entre los que podemos señalar los siguientes (Lin, 2003): la facilidad del acceso a la información, una mejor coordinación de las actividades, una mayor facilidad para la toma de decisiones colectivas o la reducción de los costes de transacción.

\subsubsection{Desde la perspectiva de la empresa}

Para analizar la calidad macroeconómica del factor empresarial consideraremos a la empresa desde una perspectiva agregada, es decir, se tendrán en cuenta los rasgos de la estructura o tejido empresarial y su vínculo con el territorio. Existen diversos rasgos que definen esa calidad, siendo los más relevantes el tamaño empresarial, la especialización productiva del territorio, la dependencia productiva y la dependencia funcional.

El tamaño empresarial hace referencia a la dimensión de una empresa. Aunque los indicadores que se utilizan para medir el tamaño empresarial son diversos, se suele utilizar el número de empleados que tiene la empresa (Camisón, 2001). La relación del tamaño empresarial y el desarrollo económico fue reconocida por primera vez por Schumpeter (1934), cuando afirmó que las grandes empresas eran las principales responsables de las innovaciones y del progreso tecnológico debido a que estaban en mejores condiciones competitivas por su mayor infraestructura financiera, de marketing y de I+D. No obstante, a partir de los años setenta las PYMES se empezaron a considerar unidades productivas de gran relevancia en los 
procesos de desarrollo económico, aunque las grandes empresas siguen teniendo gran presencia en los territorios desarrollados $\mathrm{y}$, por tanto, su papel sigue siendo muy destacado en este mundo de la globalización (Fernández y Romero, 2013).

Respecto al sector productivo dominante, cabe señalar que aunque el sector industrial en su conjunto constituya un sector de gran importancia por los efectos de arrastre que genera en otras actividades productivas, es la industria de alto contenido tecnológico la que más contribuye al desarrollo económico junto con los servicios intensivos en conocimientos. Como consecuencia de ello, una estructura empresarial con una mayor presencia de empresas en estas actividades tendría mayor calidad desde la perspectiva macroeconómica que se considera en este trabajo (Santos et al., 2012).

Por su parte, la dependencia o autonomía funcional es un concepto reciente desarrollado por Guzmán et al. (2009) para definir la calidad del tejido empresarial de un territorio. Una empresa presenta una dependencia funcional extrema cuando realiza todas sus compras fuera del territorio y destina todas sus ventas al mercado local. Por el contrario, una empresa con una autonomía funcional extrema comprará todos sus inputs en el mercado local y venderá la totalidad de sus productos terminados en otros mercados (Guzmán et al., 2009). Una empresa completamente autónoma desde el punto de vista funcional contribuirá más al desarrollo económico local que una que tenga una dependencia funcional alta, ya que los encadenamientos hacia atrás tendrán lugar en el territorio a través de las compras de recursos endógenos, los cuales generarán efectos de arrastre. Pero al mismo tiempo, una empresa con una completa autonomía funcional vendería todos sus productos en los mercados externos gracias a la participación en las cadenas de valor global. Ello permitiría a la empresa contribuir aún más al proceso de desarrollo económico a través de la extracción de excedentes desde el exterior hacia el territorio y el reforzamiento de la capacidad competitiva de la empresa.

Por último, la dependencia productiva hace referencia al nivel de concentración de inputs y outputs de una empresa respecto a un mayor o menor número de proveedores y clientes. Se considerará que existe una alta dependencia productiva cuando los inputs o outputs de una empresa se encuentran concentrados en un reducido número de proveedores o clientes. Por el contrario, se hablará de una alta autonomía productiva cuando los inputs o outputs de una empresa se encuentran distribuidos entre un gran número de proveedores $\mathrm{o}$ clientes (Guzmán et al., 2009). Una dependencia productiva alta tanto de clientes como de 
proveedores colocaría a la empresa en una clara situación de desventaja, ya que ésta vería limitada su capacidad de negociación, quedando subordinada a las decisiones de otras empresas (Porter, 1985).

\section{MODELO TEÓRICO DE ENLACE}

Tras la revisión de la teoría del entrepreneurship cabe preguntarse varias cuestiones: ¿Cuál es la calidad del factor empresarial cooperativo? ¿Muestran sus empresas la misma calidad que las empresas convencionales? ¿Se diferencian los dos modelos empresariales en alguna de las variables señaladas anteriormente? De ser así, ¿en cuáles y en qué medida? La literatura existente sobre cooperativas a este respecto es muy escasa y en ocasiones contradictoria y poco concluyente. No obstante, existen algunas aportaciones que nos pueden permitir plantear ciertas hipótesis en determinadas dimensiones de la calidad empresarial. Por tanto, antes de proponer un modelo teórico que enlace las dos líneas de investigación mencionadas (cooperativas y entrepreneurship) con el desarrollo económico, se va a proceder a revisar, en primer lugar, aquellas contribuciones de la literatura que se refieren a las dimensiones de la calidad macroeconómica del factor empresarial cooperativo tanto desde la perspectiva del empresario como desde la perspectiva de la empresa.

\subsection{Calidad macroeconómica del comportamiento emprendedor cooperativo}

Comenzando por el comportamiento innovador, son varios los autores que señalan que las cooperativas tienen más dificultades para llevar a cabo innovaciones de mayor grado, principalmente por la falta de financiación (Coque, 2008; Novkovic, 2007). No obstante, también diversos estudios sostienen que los principios cooperativos facilitan la innovación a través de la cooperación y la formación, favoreciendo de esta forma el intercambio de información y la capacidad de absorción del conocimiento (Marcuello y Saz, 2008; Rodríguez y Guzmán, 2013; Gómez y Mendizábal, 2014).

En lo que se refiere a la "asunción de riesgos", la mayor parte de la literatura al respecto reconoce que las cooperativas tienen mayor aversión al riesgo que sus competidoras capitalistas (Ben-Ner, 1988b; Pérotin, 2006). Esta mayor aversión al riesgo se produce porque los cooperativistas parten de una situación de más riesgo que los empresarios convencionales, ya que concentran todos sus esfuerzos laborales -y normalmente también de capital- en una sola empresa, al contrario que los inversores capitalistas, quienes suelen distribuir sus recursos entre varias entidades para diversificar el riesgo (Drèze, 1996). 
Por su parte, la "proactividad" ha sido muy poco estudiada en las empresas cooperativas. Coll y Cuñat (2008) encuentran que las cooperativas valencianas presentan una escasa proactividad debido a que sus emprendedores asignan las tareas administrativas a asesorías externas y a que éstos tienen una gran falta de visión estratégica. Sin embargo, Charterina-Abando, Gallastegi y Rodríguez (2007) obtienen como resultado que las cooperativas vascas presentan una visión más proactiva que las empresas convencionales debido a que la gestión empresarial medida en torno a varias variables es de mayor calidad.

Con respecto a la dimensión de "ambición”, Coll y Cuñat $(2007,2008)$ concluyeron en sus estudios que las empresas cooperativas valencianas no presentaban deseos de incrementar su volumen de negocio. Si a ello sumamos que las empresas cooperativas, dada su filosofía empresarial, anteponen otros principios y valores (tales como el compromiso con el entorno, el bienestar del ser humano, etc.) al beneficio económico, parece lógico que estas empresas puedan presentar un comportamiento menos ambicioso que las empresas capitalistas.

Por último, con respecto a la "cooperación", cabe mencionar que la mayoría de los investigadores aceptan que las cooperativas están mejor equipadas que las empresas convencionales para llevarla a cabo por fundamentarse en valores de igualdad y beneficio colectivo (Coque, 2008; Bauer, Guzmán y Santos, 2012).

\subsection{La calidad macroeconómica del tejido empresarial cooperativo}

Comenzando por el factor tamaño empresarial, aunque tradicionalmente se ha asociado a las cooperativas con empresas muy pequeñas, en los últimos estudios se suelen identificar en mayor medida con pequeñas y medianas (Burdin y Dean, 2009; Fakhfakh, Pérotin y Gago, 2012). En este sentido, las empresas cooperativas cada vez se empiezan a parecer más a las empresas típicamente capitalistas en esta dimensión empresarial.

Asimismo, la literatura sobre cooperativas señala que éstas desarrollan su actividad productiva en todo tipo de sectores, aunque dependiendo de los territorios se concentran más en unos que en otros (Burdín y Dean, 2009). En consecuencia, la evidencia disponible no permitiría establecer diferencias entre las empresas convencionales y las cooperativas respecto al factor de especialización productiva.

\footnotetext{
${ }^{6}$ Cabe señalar que, en el caso de las cooperativas agrarias españolas, la cooperación es aún una tarea pendiente que podría aumentar el éxito empresarial de las mismas (Meliá y Juliá, 2008).
} 
En cuanto al factor "dependencia funcional" y teniendo en cuenta el compromiso con el entorno que tienen las empresas cooperativas (Calvo y González, 2011), se entiende que la proporción de compras procedentes del mercado local sería mayor en las cooperativas que en las empresas convencionales. Asimismo, aunque sus principios no establezcan que se deben vender los productos y servicios en el mercado local, el fuerte arraigo al territorio de las cooperativas conduciría a pensar que la proporción de las ventas destinadas al mercado local también sería mayor que en el caso de las empresas tradicionales (Bauer et al., 2012).

Por otro lado, con respecto al factor “dependencia productiva”, Coll y Cuñat (2007) encontraron que las cooperativas valencianas contaban con muy pocos clientes debido a que la mayoría de las ventas eran absorbidas por los ayuntamientos, acomodándose a ese nivel de ingresos. Este resultado, unido al hecho de que el arraigo al territorio (Tarazona y Albors, 2005) limita el número de proveedores y clientes a los existentes en el mismo, lleva a pensar que las cooperativas podrían contar con un nivel mayor de dependencia productiva que las empresas convencionales tanto por el lado de los clientes como por el de los proveedores.

\subsection{El modelo de enlace y la esencia cooperativa}

El modelo de enlace propuesto (figura 1) parte de la idea de que la calidad macroeconómica del factor empresarial de un territorio vendría determinada por la calidad del factor empresarial convencional y del cooperativo, actuando ambos de forma complementaria en su contribución al desarrollo.

Según la literatura revisada anteriormente, se podría afirmar que la calidad macroeconómica de un factor empresarial (ya sea convencional o cooperativo) estaría determinada por dos elementos: los comportamientos emprendedores del empresario y las características de su tejido empresarial. De esta forma, cuanta mayor fuese la calidad de estos dos elementos, mayores serían las posibilidades de contribución del factor empresarial al desarrollo económico del territorio (Santos et al., 2012; Fernández y Romero, 2013). Ahora bien, hay que tener en cuenta que, dada la naturaleza de los dos factores empresariales considerados, podrían existir diferencias entre ambos respecto a los dos elementos de calidad y, por tanto, sus posibilidades de contribución al desarrollo también podrían ser diferentes

Asimismo, en el caso del factor empresarial cooperativo existe un elemento adicional que actuaría como un complemento en la calidad macroeconómica de estas entidades, algo con lo que no cuentan las empresas convencionales: la "esencia cooperativa". Ésta haría 
referencia al respeto y puesta en práctica de los principios y fundamentos que caracterizan a estas entidades empresariales. La "esencia cooperativa" se podría medir mediante el grado de cumplimiento de los principios cooperativos (democracia, cooperación, etc.) y de otros rasgos específicos de las cooperativas (motivación para su creación, no existencia de proceso de degeneración, etc). Así, tal y como se deduce de la literatura revisada, cuanto mayor fuese la "esencia cooperativa", mayor posibilidad habría de que las cooperativas influyesen positivamente sobre el desarrollo del territorio tanto en términos económicos como de cohesión social (Melián y Campos, 2010; Martínez Charterina, 2015).

Figura 1. Calidad macroeconómica empresarial y desarrollo económico

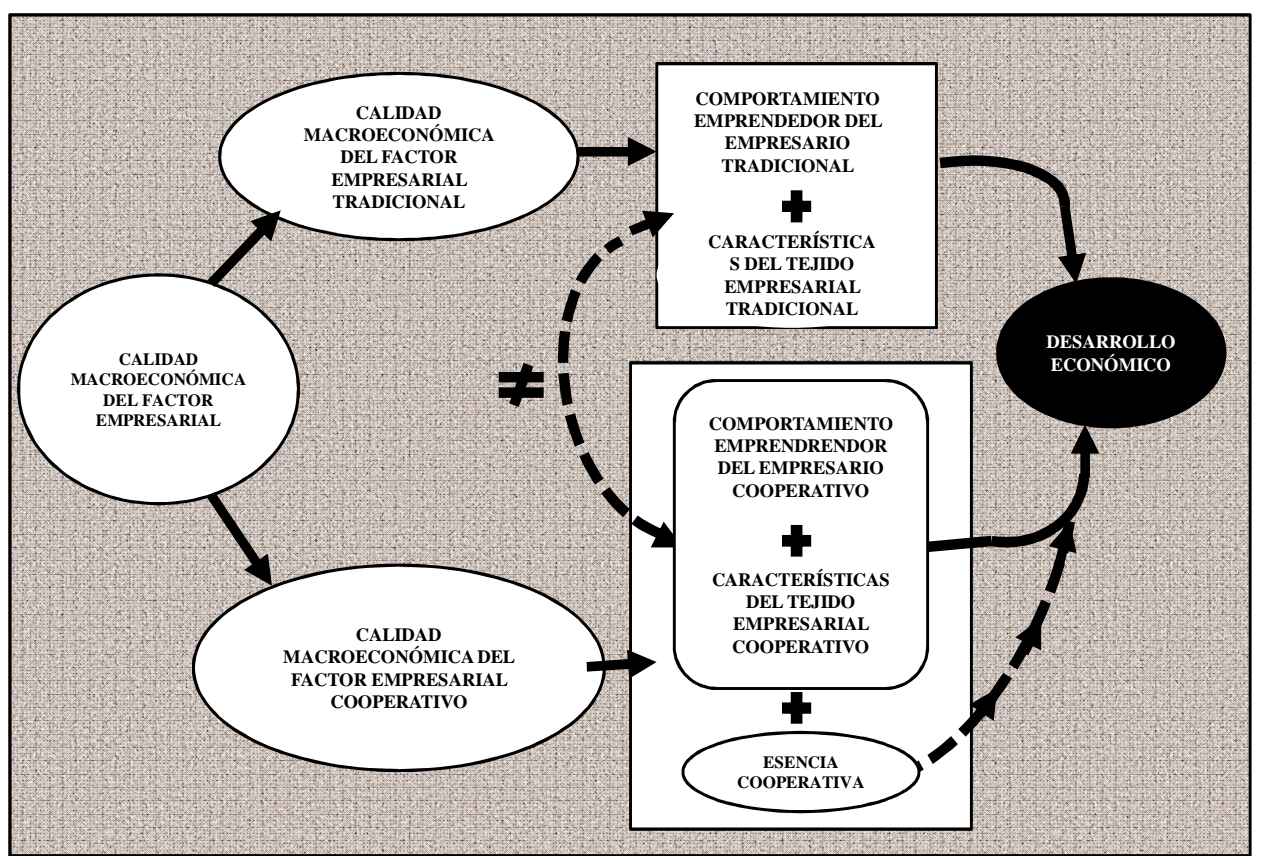

Fuente: Elaboración propia

\section{CONCLUSIONES}

Este artículo tenía como propósito analizar el papel de las cooperativas en los procesos de desarrollo económico de los territorios siguiendo el enfoque del entrepreneurship, y, más concretamente, el de la calidad macroeconómica empresarial. En base a la revisión de la literatura realizada, se ha propuesto un modelo teórico según el cual la calidad del factor empresarial cooperativo complementaría a la calidad del factor empresarial convencional en su contribución al desarrollo de un territorio a través de una dimensión adicional al comportamiento emprendedor de los empresarios y a las características del tejido empresarial (Santos et al., 2012; Fernández y Romero, 2013): la "esencia cooperativa". Ésta hace 
referencia al cumplimiento de la filosofía cooperativa y sus principios. Lógicamente, el modelo propuesto tendría que ser probado empíricamente con muestras de empresas cooperativas y convencionales de territorios de diverso grado de desarrollo a fin de contrastar su validez en la práctica. De esta forma, en función de los resultados que se obtuvieran se podrían realizar recomendaciones a los poderes públicos con el objetivo último de conseguir mayores niveles de desarrollo económico en los territorios.

Por último, una importante cuestión que cabe señalar respecto al modelo teórico propuesto es que establece como supuesto de partida una relación causa-efecto de la calidad del factor empresarial cooperativo sobre el desarrollo, aunque no debe olvidarse que en todo proceso de desarrollo siempre existe una dinámica de retroalimentación que puede conducir a plantear en este caso específico la siguiente cuestión: ¿los territorios relativamente más avanzados lo son, entre otros motivos, por la alta esencia cooperativa o, por el contrario, tienen una esencia cooperativa mayor debido a su alto nivel de desarrollo? Asimismo, esta pregunta también podría realizarse sobre los otros componentes del factor empresarial cooperativo: el comportamiento emprendedor y los rasgos del tejido empresarial. En este sentido, para dar respuesta a estas cuestiones sería necesario crear un índice de medición de los distintos aspectos de la calidad macroeconómica empresarial cooperativa y realizar estudios empíricos con datos de panel y muestras representativas de cooperativas de distintos territorios.

\section{BIBLIOGRAFÍA}

ALIANZA COOPERATIVA INTERNACIONAL (ACI) (1995) Principios Cooperativos. http://www.ica.coop/es/coop/principios.html

BAUER, C. M.; GUZMÁN, C. y SANTOS, F. J. (2012) Social capital as a distinctive feature of Social Economy firms. International Entrepreneurship and Management Journal, $\mathrm{n}^{\mathrm{o}} 8$, vol. 4, pp. 437-448.

BAUMGARTNER, D; SCHULZ, T. y SEIDL, I. (2013) Quantifying entrepreneurship and its impact on local economic performance: A spatial assessment in rural Switzerland. Entrepreneurship \& Regional Development, $\mathrm{n}^{\circ} 25$, vol. 3-4, pp. $222-250$.

BAUMOL, W. (1990) Entrepreneurship: productive, unproductive and destructive. The Journal of Political Economy, n 98, vol. 5, pp. 893-921. 
BENNER, A. (1988b) The life cycle of worker-owned firms in market economies: a theoretical analysis. Journal of Economic Behaviour \& Organization, $\mathrm{n}^{\mathrm{o}}$ 10, vol. 3, pp. 287-313.

BIRCHALL, J. (2013) The potential of co-coperatives during the current recession; theorizing comparative advantage. Journal of entrepreneurial and organizational diversity, $\mathrm{n}^{\mathrm{o}} 2$, vol. $1,1-22$.

BUENDÍA, I. y CARRASCO, I. (2014) El impacto de los factores institucionales en la actividad emprendedora: un análisis del cooperativismo europeo. Revista de economía mundial, $\mathrm{n}^{\circ}$ 38 , pp. $175-200$.

BUENDÍA, I. y GARCIA J. V. (2003) Cooperativismo y desarrollo local. Editorial Centro de Estudios Ramón Areces.

BURDÍN, G. y DEAN, A. (2009) New evidence on wages and employment in worker cooperatives compared with capitalist firms. Journal of Comparative Economics, no 37, vol. 4 , pp. $517-533$.

CALDERÓN, B. Y CALDERÓN, M. J. (2012a) La calidad del empleo de las entidades de la economía social en período de crisis. Ekonomiaz, Revista Vasca de Economía, no 79, pp. $31-57$.

CALDERÓN, B. Y CALDERÓN, M. J. (2012b) Cómo afrontan la crisis las cooperativas en España: comparativa de Trayectorias laborales a partir de la muestra continua de vidas laborales. CIRIEC-España, no 76, pp. 4-26

CALVO, R. Y GONZÁLEZ, J. (2011) La creación de empresas de economía social en el modelo de desarrollo local: reflexiones sobre la divergencia actual del caso valenciano. REVESCO. Revista de Estudios Cooperativos, $\mathrm{n}^{\circ}$ 104, pp. 7-37.

CAMISÓN, C. (2001) La investigación sobre la PYME y su competitividad: balance del estado de la cuestión desde las perspectivas narrativa y meta-analítica. Papeles de Economía Española, nº 89/90, pp. 43-83.

CANTERO, S.; GONZÁLEZ LOUREIRO, M. y PUIG, F. (2013) The "social economy" effect on business survival. Ciriec España, no 78, pp. 175-200.

CARLSSON, B. (1996) Small business, flexible technology and industrial dynamics. En ACS, Z. J.; CARLSSON, B. y THURIK, R. (eds): Small business in the modern economy. Oxford: Blackwell Publishers, pp 63-125.

CARRASCO, I. (2005) La ética como eficiencia: la responsabilidad social en las cooperativas de crédito españolas. CIRIEC-España, nº 53, pp. 351-367. 
CHARTERINA-ABANDO, J., GALLASTEGI, E. y RODRIGUEZ, J. (2007) The quality of management in Basque companies: differences existing between cooperative and noncooperative companies. Advances in the Economic Analysis of Participatory and LaborManaged Firms, nº 10 (SUPPL.), pp. 109-150.

CHAVES, R. y MONZON, J.L. (2012) Beyond the crisis: the social economy, prop of a new model of sustainable economic development. Service Business, ${ }^{\circ}$ 6, vol. 1, pp 5-26.

COLL, V. y CUÑAT, R. (2007) ¿Contribuyen las cooperativas de reciente creación al desarrollo local? Una visión desde los principios cooperativistas. Revista Venezolana de Economía Social, nº13, pp. 8-29.

COLL, V. Y CUÑAT, R. (2008) Nuevas cooperativas de trabajo asociado: un análisis del proceso de creación basado en la teoría fundamentada. Revista Venezolana de Economía Social, $\mathrm{n}^{\circ} 15$, pp. 11-36.

COMISIÓN MUNDIAL DE MEDIO AMBIENTE Y DESARROLLO (1987) Our common future. Oxford: Oxford University Press.

CORNFORTH, C. Y THOMAS, A. (1995) Les cooperatives de travail au Rouyaume Uni. Declin ou croissance? Revue de Etudes Cooperatives, Mutualistes et Associatives, $\mathrm{n}^{\mathrm{o}} 255$, vol. 53.

COQUE, J. (2008) Puntos Fuertes y débiles de las cooperativas desde un concepto amplio de gobierno empresarial. REVESCO. Revista de Estudios Cooperativos, $\mathrm{n}^{\circ}$ 95, pp. 65-93.

COVIN, J. G. y SLEVIN, D.P. (1989) Strategic management of small firms in hostile and benign environments. Strategic Management Journal, $\mathrm{n}^{\mathrm{o}}$ 10, vol. 1, pp. 75-88.

DAVIDSSON, P. (1991) Continued entrepreneurship: ability, need and opportunity as determinants of small firm growth. Journal of Business Venturing, no 6, pp. 405-429.

DEFOURNY, J. (1990) L'émergence du secteur d'économie sociale en Wallonie. Cooperatives et Développement, $\mathrm{n}^{\circ}$ 23, vol. 1, pp. 151-175.

DOH, S. y MCNEELY, C.L. (2012) A multi-dimensional perspective on social capital and economic development: an exploratory analysis. The Annals of Regional Science $\mathrm{n}^{\mathbf{0}} 49$, vol. 3, pp. 821-843.

DRÈZE, J. H. (1976) Some theory of labour management and participation. Econometrica, $\mathrm{n}^{\circ}$ 44, vol. 6, pp. 1125-1139.

ERRASTI, A. Y MENDIZÁBAL, A. (2007) The impact of globalization and relocation strategies in large cooperatives: The case of Mondragon Cooperative Fagor Electrodomésticos, S. Coop., Cooperative firms in global markets: incidence, viability and 
economic performance. Advances in the economic analysis of participatory and labormanaged firms, 10, pp. 265-295.

FAKHFAKH, F., PÉROTIN, V. y GAGO, M. (2012) Productivity, capital and labor in labormanaged and conventional firms. Industrial and Labor Relations Review, $\mathrm{n}^{\circ}$ 65, vol. 4.

FERNÁNDEZ, J. y ROMERO, I. (2013) Entrepreneurial quality and regional development: Characterizing SME sectors in low income areas. Papers in Regional Science, ${ }^{\circ}$ 92, vol. 3, pp. 495-513.

GARGALlO, A. y FREUNDLICH, F. (2010) Percepciones de los socios y no socios cooperativistas sobre la satisfacción laboral. REVESCO. Revista de Estudios Cooperativos, $\mathrm{n}^{\mathrm{o}} 103$, pp. 33-58.

GÓMEZ, J. M., y ROMAN, M. A. (2005) La Economía Social y su contribución a la promoción del desarrollo local y regional. Revista Universitaria de ciencias del trabajo, $\mathrm{n}^{\circ}$ 6, pp. 254-289.

GÓMEZ, J.M. y MENDIZÁBAL, G. (2014) Economía social y comportamiento innovador: estudio empírico de las empresas de economía social en Castilla y León. CIRIEC-España, no 81 , pp. 191-216.

GRÁVAlOS, M. A. Y POMARES, I. (2001b) Cooperativas, desempleo y efecto refugio. REVESCO. Revista de Estudios Cooperativos, $\mathrm{n}^{\circ} 74$, pp. 69-84.

GUZMÁN, J. (1994) Towards a taxonomy of entrepreneurial theories. International Small Business Journal, $\mathrm{n}^{\mathrm{o}}$ 12, vol. 4, pp. 77-88.

GUZMÁN, J. (2006) El rol del entrepreneurship en el proceso del crecimiento económico. Estudios de Economía Aplicada, nº 24, vol. 2, pp. 361-387.

GUZMÁN, J. y SANTOS, F. J. (2009) Entrepreneurial structure from a regional perspective, en Galindo, M. A.; Guzmán, J. y Ribeiro, D.: Entrepreneurship and business: a regional perspective. Berlin, Alemania: Springer, pp. 51-75.

GUZMÁN, J., CÁCERES, R. y RIBERIO, D. (2009) Functional dependence and productive dependence of SMEs. Small Business Economics, n 32, vol. 3, pp. 317-330.

HÉBERT, R. F. y LINK, A. N. (1989) In search of the meaning of entrepreneurship. Small Business Economics, $\mathrm{n}^{\circ}$ 1, pp. 39-49.

JONES, D. (2007) The productive efficiency of Italian producer cooperatives: evidence from conventional and cooperative Firms. Advances in the Economic Analysis of Participatory and Labour Managed Firms, $\mathrm{n}^{\circ}$ 10, pp.3-28. 
JONES, D. y KALMI, P. (2009) Trust, inequality and the size of the co-operative sector: cross-country evidence. Annals of Public and Cooperative Economics, no 80, vol. 2, pp. 165-195.

JONES, D. y SVENJAR, J. (eds.) (1982) Participatory and self-managed firms, Lexington, Mass: Lexington Books.

KRUSE, D. y BLASI, J. (1997) Employee ownership, employee attitudes and firm performance: a review of the evidence. En Lewin, D., Mitchell, D. y Zaidi, M. (eds.): The Human Resources Management Handbook. Greenwich: CT, Jai Press, part 1.

LEE, B. (1988) Productivity and employ ownership: The case of Sweden. Stockholm, Sweden: Trade Union Institute for Economic Research.

LIN, N. (2003) Social capital, a theory of social structure and action, Cambridge: Cambridge University Press.

LUMPKIN, G. T. y DESS G. G. (1996) Clarifying the entrepreneurial orientation construct and linking it to performance, Academy of Management Review, $\mathrm{n}^{\circ}$ 21, vol. 1, pp. 135172.

MACTCHAYA, G. C. y PÉROTIN, V. (2013) The impact of cooperative patronage: The case of National Small Holder Farmers' Association (NASFAM) of Malawi in Kasungu District. Agrekon: Agricultural Economics Research, Policy and Practice in Southern Africa, $\mathrm{n}^{\circ}$ 52, vol. 2, pp. 75-103.

MARCUELLO, C. Y NACHAR, P. (2013) La sociedad cooperativa: motivación y coordinación. Un análisis desde las teorías económicas de la empresa y la economía social. REVESCO. Revista de Estudios Cooperativos, n 110, pp. 192-222.

MARCUELLO, C. Y SAZ, I. (2008) Los principios cooperativos facilitadores de la innovación: un modelo teórico. REVESCO. Revista de Estudios Cooperativos, $\mathrm{n}^{\circ}$ 94, pp. $59-79$.

MARTÍNEZ CHARTERINA, A. (2015) Las cooperativas y su acción sobre la sociedad. REVESCO. Revista de Estudios Cooperativos, no 117, 34-49.

MCROBIE, G. (1981) Small is possible. Gran Bretaña: Abacus.

MELIÁ, E. Y JULIÁ, J. F. (2008) La intercooperación: una respuesta a las actuales demandas del cooperativismo agrario. Estudios de Economía Aplicada, n²6, vol. 1, pp. 57-88.

MELIÁN, A. Y CAMPOS, V. (2010) Emprendedurismo y economía social como mecanismos de inserción socio-laboral en tiempos de crisis. REVESCO. Revista de Estudios Cooperativos, $\mathrm{n}^{\circ} 100$, pp. 43-67. 
MENDIZABAL, A., BEGIRISTAIN, A. Y ERRASTI, A. (2005) Deslocalizaciones y empleo cooperativo. El caso de Fagor Electrodomésticos, S. Coop. CIRIEC-España, no 52, pp. 237-266.

MILLER, D. (1983) The correlates of entrepreneurship in three types of firms. Management Science, $\mathrm{n}^{\mathrm{o}}$ 29, vol. 7, pp. 770-791.

MILLER, K. L. y MONGE, P. R. (1986) Participation, satisfaction and productivity: A metaanalytic review. Academy of Management Journal, nº 29, pp. 727-753.

MINISTERIO EMPLEO Y ECONOMÍA SOCIAL (2013) Noticias del gabinete de comunicación del Ministerio de Empleo y Economía Social http://prensa.empleo.gob.es/WebPrensa/noticias/ministro/detalle/2099

MONZÓN, J. L. (Dir.) (2010) Las grandes cifras de la economía social en España, Valencia: CIRIEC.

MONZÓN, J. L. y CHAVES, R. (2012) The social economy in the European Union, comunicación de CIRIEC Internacional al European Economic and Social Committee el 2 de octubre de 2012 http://www.eesc.europa.eu/resources/docs/presentation-by-dr-mr-monzon.pdf

MOZAS, A. y BERNAL, E. (2006) Desarrollo territorial y economía social. CIRIEC-España, $\mathrm{n}^{\circ} 55$, pp. 125-140.

NOVKOVIC, S. (2007) R\&D, innovation and networking: strategies for cooperative survival, Advances in the Economics of Participatory and Labour Managed Firm. En NOVKOVIC, S. y SENA, V. (editors): Cooperative firms in global markets: incidence, viability and economic performance. Amsterdam: Elsevier Science, pp. 205-233.

OCDE (1998) Fostering entrepreneurship. Paris: OCDE.

OCDE (2003) Entrepreneurship and local economic development. Programme and policy recommendations. Paris: OCDE.

PARLAMENTO EUROPEO (2013) Resolución del Parlamento Europeo, de 2 de julio de 2013, sobre la contribución de las cooperativas a la salida de la crisis. http://www.europarl.europa.eu/sides/getDoc.do?pubRef=-//EP//TEXT+REPORT+A72013-0222+0+DOC+XML+V0//ES\#title2

PASTOR, E. (2011) El papel de la economía social como motor del cambio social y la democratización sostenible de las políticas públicas sociales en el ámbito local. REVESCO. Revista de Estudios Cooperativos, $\mathrm{n}^{\circ}$ 104, pp. 143-169.

PENROSE, E.T. (1959) The theory of the growth of the firm, New York: ME Sharpe. 
PÉROTIN, V. (2006) Entry, exit and the business cycle. Are cooperatives different? Journal of Comparative Economics, № 34, vol. 2, pp. 295-316.

PLAZA-ANGULO, J. J. (2010) Las sociedades laborales andaluzas: factores de supervivencia. Tesis Doctoral. Málaga: Universidad de Málaga.

PORTER, M. (1985) Competitive advantage: Creating and sustaining superior performance. New York: Free Press.

PUENTES, R. y VELASCO, M. M. (2009) Importancia de las sociedades cooperativas como medio para contribuir al desarrollo económico, social y medioambiental, de forma sostenible y responsable. REVESCO. Revista de Estudios Cooperativos, $\mathrm{n}^{\circ}$ 99, pp, 104129.

RAUCH, A., FRESE, M. Y UTSCH, A. (2005) Effects of human capital and long-term human resources development and utilization on employment growth of small-scale businesses: A causal analysis. Entrepreneurship Theory and Practice, $\mathrm{n}^{\mathrm{o}} 29$, vol. 6, pp. 681-698.

RODRÍGUEZ, M. J. y GUZMÁN, C. (2013) Innovation in social economy firms. Management Decision, $\mathrm{n}^{\circ}$ 51, vol. 5, pp. 986-998.

SANTOS, F. J. (2004) Convergencia, desarrollo y empresarialidad en el proceso de globalización económica. Revista de Economía Mundial, nº 10/11, pp. 171-202.

SANTOS, F. J., ROMERO, I. y FERNANDEZ, J. (2012) SMEs and entrepreneurial quality from a macroeconomic perspective. Management Decision, $\mathrm{n}^{\circ}$ 50, vol. 8, pp. 1382 - 1395.

SANTOS, F. J., BARROSO, M. O. y GUZMÁN, C. (2013) La economía global y los emprendimientos sociales. Revista de Economía Mundial, no 35, pp. 177-196.

SAZ, M. I. y CARÚS, L. (2008) Los procesos participativos en la sostenibilidad medioambiental. El caso del segmento turístico recreativo de Alta Montaña. CIRIECEspaña, $\mathrm{n}^{\circ}$ 61, pp. 127-156.

SCHUMPETER, J.A. (1934) The theory of economic development. Harvard: Harvard University Press.

STÖHR, W. B. (1984) Selective self-reliance and endogenous regional development. Preconditions and constraints, Wien: Interdisziplinares Institut fur Raumordnung.

TARAZONA, P. y ALBORS, J. (2005) La economía social y el desarrollo local. Revista Noticias del CIDEC, n 45 , pp. 70-75.

VÁZQUEZ, J.J.; ALBARRÁN, C. y SALINAS, F. (2013): The social economy and the new social welfare paradigm. Ciriec-España, nº 79, 5-34. 
VENKATRAMAN, N. (1989) Strategic orientation of business enterprises: The construct, dimensionallity, and measurement. Management Science, $\mathrm{n}^{\mathrm{o}}$ 35, vol. 8, pp. 942-962.

WARD, B (1958) The firm in Illyria: market syndicalism. American Economic Review, $\mathrm{n}^{\circ}$ 48, pp. 566-589.

WENNEKERS, S. y THURIK, R. (1999) Linking entrepreneurship and economic growth. Small Business Economics, no 131, pp. 27-55.

WESTLUND, H. y WESTERDAHL, S. (1996) Contribución de la economía social a la ocupación laboral local. Ciriec-España, n 24, pp .23-50. 